\title{
La correspondencia entre las partes del vestigio de la Trinidad y la tríada de Sap 11:21 en la obra de Juan Duns Escoto
}

Jacopo Francesco Falà

Instituto de Estudos Filosóficos, Universidade de Coimbra, Portugal

orcid.org/0000-0003-2374-4793

Recibido el 14 de febrero de 2019. Aceptado el 2 de abril de 2019.

\begin{abstract}
Resumen
El texto bíblico de Sap 11:21 tuvo una notable influencia en la historia de la filosofía medieval. Un ejemplo interesante de su importancia para la especulación teológica de la época se puede encontrar en la doctrina del vestigio de la Trinidad que Agustín elabora y configura en el sentido de una triple correspondencia entre Dios y la criatura. Esta doctrina se extenderá hasta los siglos XIII y XIV donde será central en el contexto del debate sobre el estado de las esencias creaturales, así como sobre el tema de la relación entre Dios y las criaturas. La teoría de Juan Duns Escoto será examinada en profundidad por su originalidad respecto de la tradición agustiniana y de las doctrinas de sus contemporáneos.
\end{abstract}

PALABRAS CLAVE: ESENCIA, RELACIÓN, TRINIDAD, VESTIGIO.

The Correspondence between the Part of the Vestige of the Holy Trinity and the Sap 11:21 Triad in John Duns Scotus' Work

\begin{abstract}
The biblical text of Sap 11:21 has had a notable influence on the history of Medieval Philosophy. An interesting example of its importance for the theological speculation of that time can be found in the doctrine of the vestige of the Holy Trinity, which Augustine conceive as a triple correspondence between God and creature. During the 13th and 14 th centuries this doctrine will become crucial in the context of the debate on the status of creatural essences, as well as on the issue of the relation between God and creature. The theory of John Duns Scotus will be examined in depth because of its originality in relation to the Augustinian tradition and the doctrines of Scotus' contemporaries.
\end{abstract}

KEYWORDS: ESSENCE, RELATION, HOLY TRINITY, VESTIGE.

* El siguiente texto es una traducción de Patristica et Mediaevalia del original en italiano publicado en este mimso volumen. 
La célebre sentencia bíblica según la cual Dios "ha dispuesto cada cosa según medida, número y peso" (Sap 11:21) ha sido objeto de múltiples interpretaciones, así como ha sido utilizada en varios contextos filosófico-teológicos. El presente estudio intentará indagar una de las numerosas aplicaciones de la tríada bíblica, aquella que la conecta con las partes del vestigio de la Trinidad (vestigium trinitatis), poniendo particular atención en la doctrina de Juan Duns Escoto acerca de este tema.

Para hacer esto, en primer lugar será necesario tomar en consideración el concepto de vestigio de la Trinidad, mostrando luego cómo se relaciona con los tres elementos constitutivos del orden metafísico que Dios ha impreso en la criatura: medida (mensura), número (numerus) y peso (pondus).

\section{Origen del concepto de vestigio de la Trinidad y la corresponden- cia con la tríada de Sap 11:21}

El tema del vestigio es introducido en la reflexión teológica y filosófica por Agustín. En De Trinitate establece una diferencia entre aquello que podemos conocer de la Trinidad a través de la criatura como una similitud imperfecta, en tanto "rastro", "vestigio", "huella" (vestigium) de la Trinidad misma y aquello que podemos conocer a través de la criatura como la similitud más próxima a Dios, es decir, como "imagen" (imago). Es imagen de la Trinidad solo y exclusivamente aquello que en la creación es inmortal y capaz de preservar eternamente esta similitud, esto es, la mente humana y las criaturas angélicas; ${ }^{1}$ en cambio todas las criaturas se consideran indistintamente como vestigios de la Trinidad. Las criaturas que no poseen alma racional -ya sea que se trate de criaturas inanimadas como las sustancias inorgánicas o de criaturas animadas como los animales- expresan la Trinidad solo en tanto similitudes imperfectas; por el contrario, las criaturas que poseen alma racional expresan la Trinidad también como imagen. El tema de la mente humana como imagen de la Trinidad es bien conocido en el ámbito de la psicología agustiniana y se expresa en la célebre doctrina según la cual la mente está formada por tres facultades: memoria, intelecto y voluntad, que reflejan, de acuerdo con la similitud propia de la imagen, las tres Personas divinas. En este sentido, la memoria corresponderá a la Persona trinitaria del Padre, el intelecto al Hijo y la voluntad al Espíritu Santo. ${ }^{2}$ Las criaturas que no poseen alma racional, en cambio, solo expresan la Trinidad a través de la unidad (unitas), la forma (species) y el orden (ordo) que les son propios:

Haec igitur omnia quae arte diuina facta sunt et unitatem quandam in se ostendunt et speciem et ordinem. Quidquid enim horum est et unum aliquid est sicut sunt naturae corporum ingeniaque animarum, et aliqua specie formatur, sicut sunt figurae uel qualitates corporum ac doctrinae uel artes animarum, et ordinem aliquem petit aut tenet, sicut sunt

\footnotetext{
1 Agustín, De Trinitate XIV.4.6 (CCSL 50A: 247-248): “Nec illa igitur trinitas quae nunc non est, imago Dei erit; nec ista imago Dei est, quae tunc non erit: sed ea est inuenienda in anima hominis, id est rationali, siue intellectuali, imago Creatoris, quae immortaliter immortalitati eius est insita. Nam sicut ipsa immortalitas animae secundum quemdam modum dicitur; habet quippe et anima mortem suam, cum uita beata caret, quae uera animae uita dicenda est; sed immortalis ideo nuncupatur, quoniam qualicumque uita, etiam cum miserrima est, numquam desinit uiuere: ita quamuis ratio uel intellectus nunc in ea sit sopitus, nunc paruus, nunc magnus appareat, numquam nisi rationalis et intellectualis est anima humana; ac per hoc si secundum hoc facta est ad imaginem Dei quod uti ratione atque intellectu ad intellegendum et conspiciendum Deum potest, profecto ab initio quo esse coepit ista tam magna et mira natura, siue ita obsoleta sit haec imago, ut pene nulla sit, siue obscura atque deformis, siue clara et pulchra sit, semper est".

2 Agustín, Epistulae CLXIX.2.5-6 (PL 33: 744-745): "Proinde in unum Deum, Patrem et Filium et Spiritum Sanctum firma pietate credamus; ita ut nec Filius credatur esse qui Pater est, nec Pater qui Filius est, nec Pater nec Filius qui utriusque Spiritus est. Nihil putetur in hac Trinitate temporibus locisve distare; sed haec tria aequalia esse et coaeterna, et omnino esse una natura: non a Patre aliam, et a Filio aliam, et a Spiritu Sancto aliam conditam esse creaturam; sed omnia et singula quae creata sunt vel creantur, Trinitate creante subsistere [...]. Ad hoc utcumque intellegendum assumimus memoriam, intellegentiam, voluntatem. Quamvis enim haec suis separatisque temporibus singillatim singula enuntiemus, nihil tamen horum sine aliis duobus agimus aut dicimus".
} 
pondera uel conlocationes corporum atque amores aut delectationes animarum. Oportet igitur ut Creatorem per ea quae facta sunt intellecta conspicientes Trinitatem intellegamus cuius in creatura quomodo dignum est apparet uestigium. In illa enim Trinitate summa origo est rerum omnium et perfectissima pulchritudo et beatissima delectatio (Agustín, De Trin. VI.10.12: 242).

Para Agustín, por lo tanto, son estos tres elementos ontológicamente constitutivos de cada criatura los que representan a las tres Personas divinas según una similitud imperfecta: la unitas, sin la cual ninguna criatura podría ser algo individual, es un vestigio de la Persona del Padre en tanto origen (origo) del cual derivan todas las criaturas; la species, sin la cual ninguna criatura podría tener una forma definida, es un vestigio de la Persona del Hijo en tanto belleza (pulchritudo), esto es, en tanto modelo ejemplar del cual las criaturas son copias imperfectas; el ordo, sin el cual las criaturas no tendrían un fin hacia donde dirigirse, es un vestigio de la Persona del Espíritu Santo en tanto gozo (delectatio), es decir, en tanto fin último.

Esta doctrina agustiniana llama la atención de los pensadores de la Escolástica especialmente a través de la mediación del Liber Sententiarum. En la obra lombardiana, que fue profundamente influenciada por la enseñanza agustiniana, ${ }^{3}$ se reproduce fielmente la doctrina del vestigium con una ligera reelaboración que determinará el esquema clásico de correspondencia con el cual todos los pensadores medievales se enfrentarán: dejando sin cambios las parejas unitas/origo y species/pulchritudo, el ordo está directamente relacionado con la característica de la bondad (bonitas) propia del Espíritu Santo. ${ }^{4}$

Este modelo de De Trinitate, retomado por Pedro Lombardo, está acompañado por otros que interpretan con ligeras diferencias los elementos ontológicamente constitutivos de la criatura en relación con la Trinidad. Uno de estos es precisamente el que se encuentra en el célebre pasaje de Sap 11:21, objeto de esta contribución que, además, es cuidadosamente comentado por el mismo Agustín (cf. Beierwaltes, 1969: 51-61). La tríada bíblica "medida, número y peso" es fácilmente comparable a la de "unidad, forma y orden" del De Trinitate, del cual se desprende evidentemente la siguiente inspiración: a la unidad (unitas) le corresponderá la medida (mensura), a la forma (species) el número (numerus) y al orden (ordo) el peso (pondus). ${ }^{5}$

\section{La definición de vestigio y la relación "vestigial" entre Dios y la criatura en los autores de la Escolástica del siglo XIII}

Los pensadores de la Escolástica del siglo XIII profundizarán y problematizarán esta doctrina agustiniana. En primer lugar, se centrarán más ampliamente en la definición de vestigio y su distinción en relación con la de imagen. De hecho, parecería difícil

3 Sobre las fuentes del Liber Sententiarum y en particular sobre la influencia agustiniana, cf. Rosemann 2007: 137-183.

4 Pedro Lombardo, Sententiae I, d. 3, c. 1: 70-71: "Nunc restat ostendere utrum per ea quae facta sunt aliquod Trinitatis indicium vel exiguum haberi potuerit. De hoc Augustinus in libro sexto De Trinitate ait: [...]. Summa autem origo, ut Augustinus ostendit in libro De Vera Religione, intelligitur Deus Pater, a quo sunt omnia, a quo Filius et Spiritus Sanctus. Perfectissima pulchritudo intelligitur Filius, scilicet veritas Patris nulla ex parte ei dissimilis, quam cum ipso et in ipso et in ipso Patre veneramur; quae forma est omnium quae ab uno facta sunt et ad unum referuntur. Quae tamen omnia nec fierent a Patre per Filium, neque suis finibus salva essent, nisi Deus summe bonus esset, qui et nulli naturae quae ab illo bona esset invidit, et ut in bono ipso maneret, alia quantum vellet, alia quantum posset, dedit. Quae bonitas intelligitur Spiritus Sanctus, qui est donum Patris et Filii".

5 En otros textos, Agustín sustituye el término "mensura" con "modus", conceptos en definitiva totalmente equivalentes. Véase Agustín, De natura boni 3 (CSEL 252: 857): "Nos enim catholici Christiani Deum colimus a quo omnia bona sunt, seu magna, seu parva; a quo est omnis modus, seu magnus, seu parvus; a quo omnis species, seu magna, seu parva; a quo omnis ordo, seu magnus, seu parvus. Omnia enim quanto magis moderata, speciosa, ordinata sunt, tanto magis utique bona sunt; quanto autem minus moderata, minus speciosa, minus ordinata sunt, minus bona sunt. Haec itaque tria, modus, species et ordo, ut de innumerabilibus taceam quae ad ista tria pertinere monstrantur, haec ergo tria, modus, species, ordo, tamquam generalia bona sunt in rebus a Deo factis, sive in spiritu, sive in corpore". 
discernir entre estos dos diferentes modos en los que la Trinidad puede ser representada: en particular, uno podría preguntarse si aquellas cosas que son imágenes de la Trinidad, como los ángeles, pueden ser también vestigios, dado que vestigio e imagen parecen ser caracterizados como rationes repraesentandi opuestas, $\mathrm{y}$, por tanto, aparentemente no parecerían ser compatibles al interior de un único sujeto.

En este sentido, la estrategia de definición más comúnmente utilizada es aquella que, a partir de Tomás, comienza a tomar en consideración el significado común de vestigium, esto es, el significado generalmente aceptado y aplicable a las sustancias corpóreas, para después predicarlo de acuerdo con una cierta similitud al vestigium como modo en el cual la Trinidad puede ser conocida a través de las criaturas.

En latín medieval, el término "vestigium" indica sobretodo un "rastro", en el sentido, por ejemplo, de la pisada dejada por el paso de un pie o una pata. Al igual que a partir de una huella es posible rastrear e individualizar el animal que pasó, del mismo modo es posible identificar la Trinidad a través de la huella, en sentido metafórico, que Dios ha dejado en la criatura creándola. Sin embargo, debe notarse que resulta difícil realizar una indagación que quiera rastrear no solo la especie a la que pertenece el animal que pasó, sino también el animal en su individualidad determinada a partir de una simple huella dejada en el terreno. De hecho, la huella no representa al animal individual en su totalidad, sino que es exclusivamente el modelo de la planta de su pata: ¿cómo es posible, a partir de un indicio así de parcial, llegar a una conocimiento que sea capaz incluso de mostrar la diferencia entre ese animal en particular y otros de la misma especie?

vestigium, secundum quod hic sumitur, metaphorice accipitur, et sumitur ad similitudinem vestigii proprie dicti, quod est impressio quaedam, confuse ducens in cognitionem alicujus, cum non repraesentet ipsum nisi secundum partem, scilicet pedem, et secundum inferiorem superficiem tantum (Tomás, Super Sent. I, d. 3, q. 2, a. 1: 100).

Si alguien quisiera identificar al animal que ha dejado la huella, para comprender aproximadamente de qué tipo de animal se trata quizás la huella (vestigium) será suficiente, pero en el caso en que se preguntara con más precisión cuál es el individuo específico de esa especie determinada, será necesaria también una fotografía, o de manera metafórica, una imagen (imago) que, en nuestro caso, se expresa solo y exclusivamente a través de alguna criatura en particular como los ángeles y las almas racionales.

Un autor influenciado por el tomismo como Egidio Romano justificará la posibilidad de esta transferencia de significado del vestigio en el sentido de huella al vestigio en el sentido de vestigio de la Trinidad expresado por las criaturas a través de la autoridad de Aristóteles, quien afirma en los Tópicos que quien se expresa metafóricamente realiza una transposición según una cierta similitud (cf. IV.2.140a 10-11). Al especificar el modo en el que es posible traer al conocimiento aquello que es buscado a través del vestigio, Egidio establece también la fórmula de la "conveniencia según la especie": el objeto es representado por el vestigio según la especie a la cual pertenece. Por ejemplo, la pisada de un ciervo dejada sobre la tierra representará a ese ciervo no simplemente por el hecho de ser un animal cualquiera (representación según el género), ni por el hecho de ser un determinado ciervo distinto en su individualidad de todos los otros (representación según el individuo) sino que, más bien, esa pisada, en tanto vestigio, representará al ciervo desde el punto de vista de su ser un ciervo cualquiera (representación según la especie).

ut scribitur VI Topicorum: "omnes transferentes secundum aliquam similitudinem transferant”; cum igitur vestigium Dei in creatura dicatur translative, non oportet 
secundum omnem modum habere rationem vestigii. Propter quod advertendum quod vestigium proprie est pedis, ad cuius similitudinem triplici via sumitur creatura respectu Dei, ut vestigium respectu animalis. Prima via sumitur ex repraesentatione: nam vestigium repraesentat personam hominis, vel alterius animalis, cuius existit medio modo inter distinctum et confusum. Nam respectu individui quaedam sunt omnino confusa, ut quae conveniunt ei ratione generis, utputa quae conveniunt Petro ratione animalis. Quaedam sunt omnino distincta, ut quae conveniunt ei ratione suae personae. Quaedam sunt medio modo, ut quae conveniunt ei ratione hominis, sive speciei. Vestigium non ducit in cognitionem omnino distinctam, quia vestigium Sortis non ducit in cognitionem Sortis, nec in cognitionem omnino confusam, sed in cognitionem medio modo, ut in cognitionem quia homo (Egidio Romano, In I Sent., d. 3, p. 1, q. 2, a. 1: 74b-75a).

Si bien no es posible indagar acerca de un individuo determinado a través del vestigio, esto es, saber de qué ciervo individual se trata en relación a los otros representantes de la misma especie, sin embargo es alcanzable un "conocimiento intermedio" que consiste en la individualización de la especie a la que pertenece el animal que ha dejado la huella. ${ }^{6}$

Luego de comprobar y definir el campo de aplicación y la forma de representar del vestigio en relación con el de la imagen, por lo general se sigue en las exposiciones de los comentarios a las Sentencias el tratamiento concerniente a la relación "vestigial" entre Dios y la criatura, a raíz de la correspondencia mencionada que traza Agustín entre las partes del vestigio y las Personas trinitarias.

La medida de la similitud que justifica la transferencia del significado del vestigio como pisada al vestigio en su sentido teológico es utilizado por los autores de la Escolástica para dar cuenta también de la relación entre la criatura en tanto vestigio y Dios. En los autores del siglo XIII esta correlación de carácter teológico está acompañada por una consideración más marcadamente filosófica respecto a los tipos de relación y a los tipos de causa implicadas en esta relación. Además, también aquí, como se dio con la definición de vestigio, se busca precisar más detalladamente el ámbito de la relación "vestigial" con respecto a la imagen entre Dios y la criatura.

Ya con Buenaventura de Bagnoregio vemos una notable extensión de esta discusión. En primer lugar, relaciona la tríada de las partes constitutivas del vestigio (unidad, forma y orden) tanto con los tres trascendentales - uno, verdadero y bueno- como con los tres tipos de causa -eficiente, formal y final. A la Persona trinitaria del Padre le corresponderá el trascendental "uno" y la causalidad eficiente; a la Persona trinitaria del Hijo el trascendental "verdadero" y la causalidad formal; a la Persona trinitaria del Espíritu Santo el trascendental "bueno" y la causalidad final. ${ }^{7}$

El Doctor Seráfico distingue asimismo con exactitud vestigio e imagen en base al tipo de conocimiento acerca de Dios que se puede rastrear a través de la criatura: el vestigio puede conducir al conocimiento de lo que es propio, esto es, a la noción de Dios en tanto causa eficiente, formal y final; en cambio la imagen puede conducir más precisamente al conocimiento de las cosas propias en tanto propias. ${ }^{8}$

6 Esta doctrina puede encontrarse en términos generales también en la tradición de pensamiento franciscano incluso en algunos autores que, como Ricardo de Mediavilla, asimilan la representación "vestigial" según la especie a aquella según el género. Ricardo de Mediavilla, Super Sent. I, d. 3, p. 1, a. 3, q. 1:45b: "Quantum ad primum, sciendum quod vestigium est impressio facta aliqua parte, et maxime pedibus animalis transeuntis per quam investigatur via incessus eius, non individuum animalis, sed genus vel speciem debiliter repraesentans, et quia repraesentatio non secundum individuum, sed secundum genus vel speciem est confusa, ideo tractum est nomen vestigium ad significandum similitudinem cuiuscumque rei ipsam confuse et debiliter repraesentantem".

7 Buenaventura, Comm. in I l Sent. d. 3, p. 1, a. un., q. 2 (Opera Omnia 1: 73b): “Nam creaturae dicuntur [...] vestigium quantum ad proprietatem, quae respicit Deum sub ratione triplicis causae, efficientis, formalis et finalis, sicut sunt unum, verum et bonum".

8 Ibid.: "Nam creatura ut umbra ducit ad cognitionem communium, ut communia; vestigium in cognitionem communia, ut 
La determinación del modo de representación del vestigio como capaz de conducir a lo que es propio en relación con las Personas trinitarias, así como la referencia a los tres tipos de causa, las encontraremos como constantes en la reflexión sobre el vestigio en los autores del siglo XIII, mientras que son fácilmente localizables variaciones y diferentes graduaciones respecto a la constitución del vestigio ya que, por lo general, se abandona la simetría buenaventuriana de los trascendentales.

Egidio Romano, por ejemplo, retoma y precisa el tema de la distinción entre lo que es propio de Dios que es posible alcanzar a través del vestigio de la Trinidad y cosas propias que es posible alcanzar a través de la imagen. ${ }^{9}$ El hecho de que el vestigio represente la Trinidad en referencia a aquello que es apropiado para las Personas trinitarias significa que representa sus atributos propios, pero no constitutivos. Por una parte, es una forma de representación más adecuada respecto a aquella meramente común, que representa en forma máximamente general y vaga; por la otra, más accidental respecto a aquella propia en el sentido pleno que, al contrario, indica la característica esencial de cada Persona, como la paternidad para el Padre y la filiación para el Hijo. En cambio, cosas apropiadas (appropriata) para las Personas son consideradas la Potencia para el Padre, la Sabiduría para el Hijo y la Bondad para el Espíritu Santo. ${ }^{10}$

Después, otros autores retoman y profundizan también la doctrina de la correspondencia entre las partes del vestigio y los tres tipos de causa, ausente en cambio en los tratamientos de Tomás de Aquino y de Egidio Romano. ${ }^{11}$ En particular, Enrique de Gante, sobre la base de una aceptación de fondo de la consideración de la representación "vestigial" como representación de las cosas apropiadas para las Personas trinitarias, proporciona un cuadro completo de esta simetría: a la medida en cuanto representante de la Potencia, característica apropiada del Padre, le corresponde la causalidad eficiente; a la forma, en cuanto representante de la Sabiduría, característica apropiada del Hijo, le corresponde la causalidad formal; al orden, en cuanto representante de la Bondad, característica apropiada del Espíritu Santo, le corresponde la causalidad final.

Et primo modo sumitur una appropriatio secundum triplex genus causae quo Deus se habet ad creata, scilicet, efficientem, formalem et finalem: et est coordinatio appropriatorum quarta, videlicet ex quo omnia patri, et hoc secundum genus causae efficienti, per quem omnia filio, secundum genus causae formalis, artis scilicet et potentiae operativae: sic enim arte et sapientia patris procedente ab ipso pater omnia operatur per filium, ex quo omnia spiritui sancto, secundum genus causae finalis. Et appropriatur ly ex ipso patri sicut potentia; per ipsum, filio sicut sapientia; in ipso, spiritui sancto sicut bonitas. [...] Secundum vero correspondentiam partium vestigii est coordinatio appropriatorum sexta, scilicet modi, speciei et ordinis, scilicet numeri, mensurae et ponderis, iuxta illud quod expositum est in praecedenti quaestioni, ut modus et mensura approprientur patri, species et numerus filio, ordo et pondus spiritui sancto (Gante, Summa, a. 72, q. 4).

appropriata; imago in cognitionem propriorum, ut propria".

9 En esto, sin embargo, siguiendo la enseñanza de Tomás de Aquino, en STh I, q. 45, a. 7 (Opera Omnia 4: 233b): “Ad primum ergo dicendum quod repraesentatio vestigii attenditur secundum appropriata: per quem modum ex creaturis in Trinitatem divinarum Personarum veniri potest".

10 Egidio Romano, In I Sent., d. 3, p. 1, q. 2, a. 1: 75a: "Quia quaedam omnino sunt ibi communia, ut essentialia non appropriata, ut essentia, Divinitas et huiusmodi; quaedam sunt omnino propria, ut constitutiva personarum, sicut Paternitas, Filiatio et huiusmodi; quaedam sunt medio modo, ut essentialia appropriata, sicut Potentia, Sapientia et huiusmodi".

11 La correspondencia entre las partes del vestigio y los tres tipos de causa también se apoya en el ambiente franciscano. En particular, me parece interesante la posición de Ricardo de Mediavilla en Super Sent. I, d. 3, p. 1, a. 3, q. 1: 45b-46a. La opinión de Ricardo parece haber tenido una influencia considerable en el ambiente franciscano oxoniense donde se formó Escoto, dado que fue citado y tomado casi literalmente por Robert Cowton, un hermano y colega de Escoto en Oxford. En particular, parece evidente el préstamo textual en lo que concierne a la exposición de los tres artículos de vestigio (cf. Apéndice). Intenté mostrar la identidad de los puntos de vista entre Ricardo de Mediavilla y Robert Cowton sobre el vestigio de la Trinidad en mi tesis doctoral. (cf. Falà, 2017: 34-35). 
En el contexto de la filosofía de Enrique esta correspondencia con los tres tipos de causa parecería implicar otra, la de los tres tipos de relaciones indicadas por Aristóteles en Metafisica V.15. En base a la doctrina aristotélica allí expuesta las cosas pueden ser en relación con ellas mismas o según el modo de la proporción, "como el doble respecto a la mitad o el triple respecto a la tercera parte"; o según el modo de actuar y del padecer, "como lo que puede calentar en orden a lo que puede ser calentado, y lo cortante en orden a lo cortable, y, en general, lo activo en orden a lo pasivo"; o, finalmente, "según el modo de lo medible en orden a la medida, lo cognoscible respecto al conocimiento o lo sensible respecto a la sensación" (cf. Metafísica V.15.1020b 26 - 1021b 11). Al primer modo Enrique le hace corresponder el tipo de causa formal, al segundo el tipo de causa eficiente y al tercero el tipo de causa final (cf. Gante, Quodlibet IX, q. 1).

\begin{tabular}{l|l|l}
\hline $\begin{array}{l}\text { Medida (Mensura) } \\
\text { Unidad (Unitas) } \\
\text { Modo (Modus) }\end{array}$ & $\begin{array}{l}\text { Número (Numerus) } \\
\text { Forma (Species) }\end{array}$ & $\begin{array}{l}\text { Peso (Pondus) } \\
\text { Orden (Ordo) }\end{array}$ \\
Uno (trascendental) & Verdadero & Bien \\
Causalidad eficiente & Causalidad formal o ejemplar & Causalidad final \\
Potencia (característica apropiada) & Sabiduría & Bondad \\
Origen (Origo) & Belleza (Pulchritudo) & Gozo (Delectatio) \\
Padre (Persona trinitaria) & Hijo & Espíritu Santo \\
\hline
\end{tabular}

\section{Juan Duns Escoto acerca del vestigio de la Trinidad y de la corres- pondencia entre las partes del vestigio y la tríada de Sap 11:21}

Juan Duns Escoto aborda la cuestión del vestigio en el lugar habitual de la tercera distinción del primer libro, en los dos comentarios a las Sentencias que produjo en el curso de su carrera académica. Los dos textos son similares en cuanto a la estructura y en las soluciones; sin embargo, interesa mayormente a los fines de nuestra discusión aquello que encontramos en la Ordinatio. En ella, contrariamente a aquello que aparece en la Lectura, el texto de Sap 11:21 se cita explícitamente y se relaciona con los pares del vestigio. Esta cita es tanto más significativa ya que resulta ser la única en toda la obra de Escoto. Solo hay otra referencia a un pasaje bíblico, aunque implícito, en el Comentario a la Metafisica y, luego, en un contexto completamente diferente como el de la discusión sobre la intensidad de las formas. ${ }^{12}$

A propósito de la definición de vestigio, Escoto produce una doctrina original y ampliamente innovadora que parte de una crítica detallada a la opinión común generalmente aceptada por los teólogos del siglo XIII, en base a la cual sostiene que el vestigio representa en modo confuso a la especie, mientras que la imagen en un modo determinado al individuo. En su propia exposición de esta opinión Escoto parece tener en mente especialmente a Egidio Romano. De hecho, tanto Egidio como la opinión expresada por Escoto sostienen que el vestigio en sentido propio concierne a las sustancias corpóreas, pero el significado primario de "vestigio" puede ser aplicado a las cosas divinas, esto es, al modo de representación de la Trinidad a través de la criatura, a través de una cierta similitud. Cabe señalar, en particular, la presencia de la cita aristotélica tomada de Tópicos: "todos los que se expresan metafóricamente, lo hacen a través de una cierta similitud", que ya hemos encontrado en Egidio Romano, ${ }^{13}$

12 Duns Escoto, Quaest. sup. lib. Met. Arist. VIII, qq. 2-3, n. 195 (Opera Philosophica 4: 469): "De quinto: sicut creaturae procedunt a Deo ut numeri et ordinate, ita inaequaliter. Quia multae, 'numerus'; quia inaequales, 'mensura'; quia compositae, 'pondus', id est, ad dissolutionem inclinatio. Igitur maior haec illa. Sed inter se comparatio propria: ad Deum comparatio excessiva".

13 Egidio Romano, In I Sent., d. 3, p. 1, q. 2, a. 1: 74b: " ut scribitur VI Topicorum: "omnes transferentes secundum aliquam similitudinem transferant"; cum igitur vestigium Dei in creatura dicatur translative, non oportet secundum omnem modum 
y que destaca cómo la transposición de significado del vestigio en sentido común al vestigio en sentido técnico sea considerado posible justamente gracias al uso de la medida de la similitud (ratio similitudinis). ${ }^{14}$

Escoto, al romper de algún modo la continuidad de la tradición sobre el tema, discute esta definición al introducir distintos criterios para explicar la definición de vestigio, también en relación con la distinción respecto a la definición de imagen. De hecho, según el Doctor Sutil el criterio para discernir vestigio e imagen no consiste en la distinción entre representación confusa según la especie y la representación determinada según el individuo sino en la distinción entre representación según el todo y representación según la parte: el vestigio es desde su punto de vista una representación de una parte del objeto, mientas la imagen es una representación del objeto en su totalidad. Así, por ejemplo, en el caso del ciervo, la huella dejada en el terreno será vestigio de todo el animal puesto que representa solo una parte, mientas que será imagen de la planta de la pata del animal en tanto la representa en su totalidad:

vestigium est similitudo partis animalis, a qua imprimitur in aliquid sibi cedens. Sed similitudo expressa partis non est similitudo expressa "totius", quia neque secundum rationem "totius" in se, neque etiam qua 'totum' immediate cognoscitur, sed tantum arguitive, et ex hoc quia cognoscitur illud repraesentatum esse aliquid istius (Duns Escoto, Ord., I, d. 3, p. 2, q. un.: 178-179).

Una tesis que parece completamente similar a aquella ya presente muchas décadas antes de Buenaventura. La expone en una objeción a la propia opinión en el Comentario a las Sentencias, refutándola a continuación en base a la consideración según la cual Dios, siendo absolutamente simple, no puede ser representado según la parte:
Alii dicunt quod vestigium dicitur quia repraesentat secundum partem, sed imago secundum totum. Sed haec iterum differentia non valet, quia, cum Deus sit simplex, non habet repraesentans secundum partem; cum iterum sit infinitus, a nulla omnino creatura, etiam a toto mundo non potest repraesentari secundum totum (Buenaventura, In I Sent., d. 3, p. 1, art. un., q. 2: 73a-73b).

A partir de aquí se podría suponer que Escoto, para su propia elaboración doctrinal, se refiere a una tradición alternativa respecto a aquella frecuentada por los autores que consideramos anteriormente. Sin embargo no hemos podido identificarla, de la misma manera en la cual no fue posible para los Hermanos Quaracchi, editores del Commentario buenaventuriano, referir las fuentes de la opinión informada. Con todo, se debe subrayar que Escoto no presenta jamás esta posición como derivada de otros Maestros, antiqui o moderni: queda entonces abierta también la hipótesis de una coincidencia puramente casual entre las opiniones del Doctor Sutil y aquella de los alii buenaventurianos.

Resta ahora considerar la segunda parte de la exposición escotista dedicada al tema de la relación "vestigial" entre Dios y la criatura. También aquí, como a propósito de la definición de vestigio, primero se da una opinión que luego será criticada. En este caso, Escoto parecería tener en mente en particular a Enrique

\footnotetext{
habere rationem vestigii".

14 Duns Escoto, Ordinatio I, d. 3, p. 2, q. un., nn. 285-286 (Opera Omnia 3: 174-176): "In ista questione tria videnda sunt: primo -equia secundum Philosophum 'omnes transferentes secundum aliquam similitudinem transferunt'- videndum est de ratione vestigii in corporalibus, unde translatum est nomen eius ad propositum [...]. Quantum ad primum dicitur quod vestigium est impressio, derelicta ex transitu alicuius super vacuum vel plenum, ipsum imperfecte repraesentans, -et ideo 'imperfecte', quia vestigium repraesentat aliquid confuse et sub ratione speciei, imago perfecte, quia sub ratione individui: sicut per vestigium distinguitur equus a bove, vel quod transiens est equus, non bos, non autem distinguitur hic equus ab illo; sed imago distinguit, quia imago Iovis non repraesentat Caesarem".
} 
de Gante: las doctrinas de alguna manera tradicionales de la representación "vestigial" como representación de las cosas apropiadas respecto de las Personas trinitarias y la correspondencia entre las tres partes del vestigio y tres tipos de causa -ya Buenaventura, como hemos visto, había defendido estas posicionesse aproxima de hecho a la opinión de la correspondencia entre las tres partes del vestigio y los tres tipos de relación indicadas por Aristóteles que describen cada tipo de relación con Dios por parte de la criatura. Así, el primer modo de relación, según la semejanza, correspondería ex parte Dei al atributo apropiado para el Hijo, el de la Sabiduría, conectado a la causalidad ejemplar; al segundo modo, según el actuar y el padecer (que Escoto llama también según el producto y el productor), correspondería el atributo apropiado para el Padre, el de la Potencia, conectado a la causalidad eficiente; el tercer modo, según la medida, correspondería el atributo apropiado para el Espíritu Santo, el de la Bondad, conectado a la causalidad final. ${ }^{15}$

De esto deriva una doctrina no defendida especialmente por Enrique de Gante, pero presente en los autores influenciados por él como Ricardo de Mediavilla ${ }^{16}$ y Robert Cowton, ${ }^{17}$ según la cual el vestigio no es más que estas tres relaciones, las cuales no agregan nada real a la esencia de la criatura. Se trata del verdadero núcleo de la opinión expuesta, el principal objetivo sobre el que polemiza Escoto en esta cuestión.

Según el Doctor Sutil, de hecho, es ante todo falsa la idea que las criaturas tienen una triple relación con Dios. Entre la criatura y Dios está instaurada solo una relación según la medida y lo medido, o según lo que es indicado por Aristóteles como "tercer modo de la relación", ya que entre Dios y la criatura solo puede existir una relación no mutua, como es precisamente la relación del tercer tipo, que va de la criatura a Dios; nunca una relación mutua -son relaciones de este tipo las primeras dos expuestas por Aristóteles- que prevé también por lo tanto una dirección de Dios hacia la criatura. Si fueran relaciones mutuas entre Dios y la criatura resultaría inválida la autosuficiencia divina: Dios en su trascendencia no tiene necesidad de relaciones con nada, es solo y exclusivamente el término de la relación que parte de la criatura sin posible reciprocidad. ${ }^{18}$

Además y sobretodo no es cierto que el vestigio consista en meras relaciones. Al contrario, Escoto defiende una concepción de las partes del vestigio (unidad, forma y orden) como "cosas absolutas" que sirven como fundamento de la relación no mutua, instituida en un momento metafísico posterior, que conecta a la criatura con Dios. ${ }^{19}$

15 Duns Escoto, Ord.I, d. 3, p. 2, q. un., n. 287: 176: “Quantum ad secundum dicitur quod creatura habet ad Deum triplicem relationem, sicut ad triplicem causam, - et hoc secundum tres modos relativorum, quos ponit Philosophus V Metaphysicae. Quantum ad primum modum, creatura refertur ad Deum relatione fundata super 'unum', relatione scilicet 'similis', et hoc in quantum creatura est exemplata et refertur ad Deum in quantum est causa exemplaris. Quantum ad secundum modum, scilicet potentiae, refertur creatura ad Deum ut productum ad producentem. Quantum ad tertium modum, scilicet modo mensurae, refertur creatura ad Deum ut ordinata ad ipsum sicut ad causam finalem".

16 Ricardo de Mediavilla, Super Sent. I, d. 3, p. 1, a. 3, q. 1: 46a: “Quantum ad tertium, sciendum est quod ista tria, in quantum sunt partes vestigii, super creaturam, in qua non sunt, non addunt rem aliquam absolutam: ipsa enim essentia creata seipsa limitata est, ita quod ille ablativus non importat habitudinem causae efficientis, sed habitudinem per modum causae formalis, ita quod limitatio creatae essentiae super ipsam creatam essentiam non addit nisi relationem ad causam efficientem, et sua pulchritudo relationem ad causam exemplarem, et eius ordo relationem ad causam finalem, aliter enim esset modus modi, pulchritudo pulchritudinis, ordo ordinis in infinitum, aut non reperiretur in qualibet creatura vestigium Dei".

17 Robert Cowton, In Sent. I, d. 3, q. 2, (f. 34vb): "De tertio articulo sciendum quod hec tria (modus, species et ordo) non addunt super rem que est vestigium rem aliquam absolutam".

18 Duns Escoto, Ord. I, d. 3, p. 2, q. un., n. 296: 180: "Item, quod dicitur quod illi tres respectus pertinent ad tres modos relativorum, hoc videtur falsum, quia Philosophus V Metaphysicae, ponens differentiam duorum modorum ad tertium, vult quod in duobus primis modis sit mutua relatio, in tertio non, sed tantum unum dicitur ad aliud quia aliud est eius; omnis autem relatio creaturae ad Deum est non-mutua, sed tantum dicitur Deus ad creaturam quia creatura ad ipsum; ergo omnis respectus creaturae ad Deum est secundum tertium modum".

19 Ibid., 181: "Quantum ergo ad secundum articulum concedo quod omnis respectus creaturae ad Deum pertinet ad illum tertium modum relativorum. Nec in solis respectibus consistit vestigium sed in aliquibus absolutis". 
Si, de hecho, el vestigio fuera una pura relación, entonces no sería posible conocerlo antes de haber conocido todos los términos que se relacionan, ya que está en la misma naturaleza de la relación el ser posterior en el conocimiento de los términos a los que se refiere. Para el filósofo escocés, contrariamente a lo que sostuvo Enrique de Gante, la relación debe siempre presuponer los términos relacionados como cosas subsistentes: puede existir solo y exclusivamente a partir de los términos de la relación como elementos metafísicamente anteriores. Así como en el ser, también en el conocimiento: la relación puede ser conocida solo y exclusivamente a partir del conocimiento de los términos de la relación como elementos epistemológicamente anteriores. ${ }^{20}$

Ahora bien, el vestigio es, en cambio, una similitud del "vestigiado", o de lo que a través del vestigio se representa, a través del cual es posible conocer de alguna manera el "vestigiado" mismo, incluso antes que sea conocido directamente. El conocimiento del vestigio es entonces precedente al conocimiento del "vestigiado". El vínculo de anterioridad/posterioridad en el conocimiento se invierte respecto de lo que sucede en las relaciones: mientras que el conocimiento de la relación es siempre posterior al conocimiento de los términos, el conocimiento del vestigio es siempre anterior al conocimiento del "vestigiado", límite de la relación de similitud en la que el vestigio consiste. ${ }^{21}$ Sobre esta base Escoto puede concluir que la razón del vestigio no se agota solo y exclusivamente en la relación "vestigial" sino que incluye también el fundamento de este vínculo, que es algo absoluto, no reductible a una mera relación. ${ }^{22}$

Dejando a un lado el análisis en profundidad acerca de las complejas cuestiones metafísicas sobre las cuales se centra el conflicto entre Escoto y Enrique de Gante sobre el tema del vínculo entre la relación y fundamento ${ }^{23}$ llegamos a la última aclaración de Escoto sobre la relación "vestigial" a propósito de la que entra en juego el pasaje bíblico de Sap 11:21. Hemos visto cómo se cree comúnmente entre los teólogos del siglo XIII que a través del vestigio de la criatura es posible conocer lo propio de las Personas trinitarias gracias a la relación de semejanza que subsiste entre la representación "vestigial" y el objeto representado por ella. Y Escoto no solo no critica esta doctrina sino que la acepta y la hace suya ${ }^{24}$ al retomar también la correspondencia ya establecida por Buenaventura entre las partes del vestigio y los tres trascendentales: uno, verdadero y bueno. ${ }^{25}$ Sin embargo, cuestiona el modo de representación del vestigio, que generalmente se consideraba como coincidente sic et simpliciter con el modo de la semejanza, una medida que también se consideraba que presidía la transposición del significado de "vestigio" desde el sentido corpóreo al metafísico-teológico ${ }^{26}$,

20 Fedeli, 2014: 99: "Per Scoto la relazione non pone in essere qualcosa, ma collega sempre termini già esistenti". Sobre la teoría de la relación de Juan Duns Escoto, véase Henninger, 1989: 68-97. También puede consultarse en Henninger, 2013; y Beckmann, 1996.

21 Duns Escoto, Ord. I, d. 3, p. 2, q. un., n. 295: 180: "Et hoc probatur sic, quia vestigium est similitudo vestigiati, ex quo cognito cognoscitur illud, - ergo vestigium potest praecognosci naturaliter illo cuius est; sed relatio non potest praecognosci termino naturaliter; ergo etc.".

22 Ibid., 179-180: "Quod etiam dicitur in secundo membro, quod vestigium consistit in illis tribus relationibus, non videtur verum, quia licet ratio vestigii dicat respectum, quemadmodum similitudo realiter est respectus, tamen sicut non dicitur similitudo esse in respectu praecise sed in aliquo absoluto, in quo fundetur ratio similitudinis, ita etiam videtur ratio vestigii non esse in respectu praecise, sed in aliquo in quo fundatur ille respectus".

23 Acerca de las críticas que Escoto traslada a la concepción de Enrique de relación y fundamento véase Decorte, 1995: 407-429.

24 Duns Escoto, Ord. I, d. 3, p. 2, q. un., n. 300: 183: "Et sic patet in quibus in creatura consistat vestigium, et respectu quorum in divinis - quia respectu appropriatorum personis divinis".

25 Ibid., 182: "Possunt et alia multa in creaturis assignari, quae velut similia repraesentant in divinis aliqua appropriata personis, puta unum, verum et bonum: unum in creatura repraesentat unitatem appropriatam Patri, verum veritatem appropriatam Filio, bonum bonitatem appropriatam Spiritui Sancto. Et istae perfectiones omnes se habent absolute, et repraesentant absolutas perfectiones Dei appropriatas personis".

26 Ibid., 174-175: "In ista questione tria videnda sunt: primo -quia secundum Philosophum "omnes transferentes secundum aliquam similitudinem transferunt"- videndum est de ratione vestigii in corporalibus, unde translatum est nomen eius ad propositum". 
así como, en base a las reflexiones de Enrique de Gante, la primera de las tres relaciones "vestigiales", que corresponde al modo de la causalidad ejemplar operada por la Persona trinitaria del Hijo. ${ }^{27}$

En realidad -precisa Escoto- no todas las relaciones "vestigiales" son según la semejanza, esto es, no todas las partes del vestigio son "similares" a las cosas apropiadas de las Personas trinitarias que representan. De hecho, ¿en qué consisten en definitiva el modo, la forma y el orden ${ }^{28}$ o, para usar la tríada bíblica, el número, la medida y el peso que constituyen el vestigio de la Trinidad? La forma o número define la figura de la cosa, sus cualidades, sus características; el orden o peso define la finalidad de la cosa, su ser orientado hacia algo; el modo o la medida describe finalmente el límite de la cosa, su finitud, es decir, el hecho que puede ser medida. Entonces, Escoto señala cómo en Dios están ciertamente presentes las razones del orden y de la forma de las cosas pero no la razón de la medida, es decir, la región de la finitud de las cosas mismas. Mientras el orden y la forma en las cosas representan según la semejanza el orden y la forma que proviene de Dios, la medida, es decir la finitud y la limitación, representa algo totalmente diferente a lo presente en Dios, esto es, la infinitud y la ilimitación. La relación "vestigial" según la medida no será una relación de semejanza (per rationem similitudinis) sino solo de proporcionalidad (per rationem proportionalis). Para que una relación sea proporcional es suficiente solo un vínculo que no implique un carácter común bajo un mismo principio y, en cambio, sí es una condición necesaria para definir una relación de semejanza. Los ejemplos de relaciones de proporcionalidad son limitado/ilimitado y también posible/necesario, dependiente/independiente. En cambio, están en una relación de semejanza dos o más bienes, dos o más verdades, dos o más unidades. ${ }^{29}$ En otras palabras, la proporcionalidad describe una correspondencia entre cosas no homogéneas, mientras la semejanza establece una relación entre cosas que comparten el pertenecer al mismo conjunto o familia.

El vestigio también se puede designar de muchas otras maneras, tanto en aquellas cosas que representan por similitud algunas cosas apropiadas para las Personas, como por la razón de lo proporcional. De hecho, digo "representar de manera proporcional" cuando la razón de ese representante no está formalmente en Dios, sino que es algo proporcional a esa razón -como a propósito de la designación "modo, forma y orden" que parece coincidir con aquella de Sap 11: "en número, peso y medida"-. De hecho, el modo pretende ser una limitación, y, por lo mismo, es la medida en esa designación de Sap 11; el peso se considera aquí como orden y el número como forma. El número o la forma, y el peso o el orden se describen como en la primera descripción o designación, pero la medida (que es la misma aquí que el modo) no representa algo en razón de lo similar sino de lo proporcional, ya que la limitación del producto representa la limitación del productor.

\footnotetext{
27 Ibid., 176: "Quantum ad secundum dicitur quod creatura habet ad Deum triplicem relationem, sicut ad triplicem causam, - et hoc secundum tres modos relativorum, quos ponit Philosophus V Metaphysicae. Quantum ad primum modum, creatura refertur ad Deum relatione fundata super 'unum', relatione scilicet 'similis', et hoc in quantum creatura est exemplata et refertur ad Deum in quantum est causa exemplaris".

28 Escoto, para describir las partes del vestigio, utiliza en este pasaje el tema que encontramos en Agustín, De natura boni 3 (CSEL 252: 857).

29 Duns Escoto, Reportatio I-A, d. 3, q. 3, n. 79: I.206: "Secundo est videndum in quo consistit ratio vestigii et dico quod creatura non potest distincte repraesentare Trinitatem, sed quaecumque in creaturis per modum similitudinis et proportionis repraesentant appropriabilia tribus personis includunt rationem vestigii: per modum similitudinis, ut bonitas bonitatem, et veritas veritatem, et unitas unitatem; per modum proportionis, ut limitatum illimitatum, dependens independens, possibile necesse esse".
} 


\section{Conclusiones}

Con respecto al vestigio, Escoto no solo pone en discusión la teoría tradicional sobre la definición de este como representación según la especie en oposición a la definición de la imagen como representación según el individuo sino también y sobre todo es justamente en el contexto de la reflexión sobre el vestigio donde desarrolla su teoría de la relación no mutua dirigida solo y exclusivamente de la criatura hacia Dios, sin ninguna posible reciprocidad, con el fin de preservar la trascendencia divina.

En esta discusión se ubica la interpretación del pasaje bíblico de Sap 11:21 donde se aplica la distinción entre la razón de la semejanza y la razón de la proporcionalidad, utilizada justamente para evitar una consideración de la criatura demasiado próxima a la, en cambio, incomparable alteridad divina desde el punto de vista de la infinita distancia entre el límite/finitud creatural y la ilimitación/infinitud divina.

Según Escoto, la medida (mensura) o modo (modus) es ciertamente una parte del vestigio, como ya sostenía Agustín y toda una tradición de pensamiento. Sin embargo, la razón de la medida, como la razón del límite de la criatura, no puede en modo alguno estar presente en Dios, que es ilimitado y por lo tanto no "semejante" a la criatura. Su ilimitación es sobretodo representada en el vestigio creatural como limitación basada en una relación de proporcionalidad, no de semejanza, vínculo que no implica características comunes. El caso examinado relativo a la doctrina escotista acerca del vestigio de la Trinidad representa entonces una pieza adicional y significativa que consolida aquella interpretación ya ampliamente señalada en la literatura crítica que ve en Escoto uno de los más rigurosos defensores de la trascendencia divina, evidenciando esto como punto nodal de toda su construcción doctrinal. También aquí, como en otros lados, Escoto tiene cuidado en no "contaminar" el absoluto divino con determinaciones finitas y creaturales, con presuntos vínculos de imposible semejanza entre el ente infinito (Dios) y el ente finito (la criatura). También aquí, como en otros lados, el proprium de la contribución especulativa escotista es una reelaboración teológica caracterizada por la exigencia doctrinal típica del pensamiento posterior a la condena del obispo Tempier de 1277 para explicar adecuadamente la radical distinción en la naturaleza, la esencia y la acción entre estos dos polos en ningún modo reducibles a características comunes. ${ }^{30}$

30 Como se sabe, el obispo de París Étienne Tempier prohibió el 7 de marzo de 1277 la enseñanza de 219 tesis de naturaleza filosófica y teológica en la Facultad de Artes bajo su jurisdicción. Muchas de estas tesis (articuli) condenadas se refieren a doctrinas destinadas a limitar la omnipotencia divina (cf. tesis 16 a 26) o al establecimiento de relaciones de afinidad y semejanza entre Dios y las criaturas, como los ángeles (cf. las tesis de 34 a 61) o la totalidad del mundo considerado como eterno (cf. tesis de 80 a 89 ). 


\section{APÉNDICE}

La cuestión que Robert Cowton dedica al vestigio en el lugar clásico de la tercera distinción del primer libro de su Comentario a las Sentencias muestra con claridad la fuerte influencia del pensamiento de su hermano franciscano Ricardo de Mediavilla. Resulta evidente en la división de la cuestión en los tres artículos -el primero, dedicado a la definición de vestigio; el segundo, dedicado a la representación de la Trinidad a través del modo de la apropiación; el tercero, dedicado a la cuestión acerca de si las partes del vestigio son propiamente de la res que se agregan a la res que es el vestigio o si en cambio son simples relaciones con Dios- que es copia casi verbatim del texto paralelo del Comentario a las Sentencias de Ricardo. Los pilares de la opinión son: la definición de vestigio como representación según el género o la especie; la representación de la Trinidad a través del vestigio según lo que es apropiado a las Personas; la negación del estatus de res a las partes del vestigio, que no agregan entonces nada real a su fundamento, sino solo simples relaciones con Dios.

Cowton demuestra conocer también la doctrina de Escoto sobre el tema, citando en un modo muy preciso las objeciones que el Doctor Sutil expresó a la opinión sobre el vestigio que sostuvieron Ricardo y muchos otros teólogos del siglo XIII. En particular, se refiere a la crítica escotista (la cual conoce muy probablemente a partir del texto de la Lectura) según la cual es imposible que el vestigio represente según la especie y la imagen según individuo, ya que, si existiera un solo ejemplar en la especie, entonces la representación según el vestigio se superpondría a aquella según la imagen. ${ }^{31} \mathrm{Al}$ responder a esta y a otras objeciones escotistas Cowton amplía, precisa y actualiza en base a las más recientes novedades teológicas la doctrina de Ricardo, proporcionando una cierta originalidad al propio tratamiento, que por lo tanto no puede ser considerado como una mera repetición servil del pensamiento de otros.

Ricardo de Mediavilla, Super Sent. I, d. 3, p. 1, a. 3, q. 1: 45b-46a: "Quantum ad primum, sciendum quod vestigium est impressio facta aliqua parte, et maxime pedibus animalis transeuntis per quam investigatur via incessus eius, non individuum animalis, sed genus vel speciem debiliter repraesentans, et quia repraesentatio non secundum individuum, sed secundum genus vel speciem est confusa, ideo tractum est nomen vestigium ad significandum similitudinem cuiuscumque rei ipsam confuse et debiliter repraesentantem. Et quia omnis effectus gerit aliquo modo similitudinem suae causae et Deus est cuiuslibet creaturae causa; et ita quaelibet creatura aliquam similitudinem Dei gerit debiliter et confuse, maxime propter infinitam sublimitatem Dei super creaturam, ideo quaelibet creatura vestigium est creatoris per quod aliqua cognitio de creatore investigari potest secundum modum qui supra dictus est. Et haec de primo articulo. Quantum ad secundum sciendum quod modus uno modo idem est quod terminus et, quia terminatum, est limitatum et mensuratum. Ideo accipitur modus interdum pro rei limitatione vel mensura; species etiam interdum accipitur pro pulchritudine secundum quod dicitur: 'iste est valde speciosus', id est pulcher. Ex hoc autem quod Deus est causa creaturarum efficiens, exemplaris et finalis oportet in omni creatura esse limitationem eo ipso quod effecta est, et pulchritudinem eo ipso quod ad pulcherrimum exemplar facta est, et ordinem eo ipso quod finem habet: in re enim quae habet finem oportet esse ordinem ad finem. Quia ergo modus creaturae,

31 Duns Escoto, Lectura I, d. 3, p. 2, q. un., n. 219 (Opera Omnia 16: 314): “Sed contra hoc quod distinguunt repraesentationem vestigii a repraesentatione imaginis, quia 'vestigium repraesentat aliquid solum secundum speciem suam, et imago secundum propriam naturam singularem', - contra: si esset unum animal tantum, et non posset esse aliud animal, adhuc impressio pedis in pulvere esset vestigium animalis et non imago; et tamen repraesentaret singulare et non aliquid secundum speciem, sicut patet ex hypothesi. Oportet igitur dare aliam differentiam inter vestigium et imaginem, sicut post dicetur". Robert Cowton, In I Sent., d. 3, q. 2: "Contra. Si non esset nisi unum animal specie et numero, nec esse posset, adhuc impressio pedis representaret animal secundum numerum, quia simili modo representaret, tunc est unicum". 
hoc est eius limitatio vel mensura, Deum repraesentat sub ratione causae efficientis, et pulchritudo eius Deum repraesentat sub ratione causae exemplaris, et ordo ipsum repraesentat sub ratione causae finalis, quae est repraesentatio non distincta, quia non est quantum ad propria personarum, sed quantum ad appropriata, non tamen sub ratione qua appropriata sunt. Ideo in qualibet creatura dicitur consistere Dei vestigium quantum ad eius modum, id est limitationem vel mensuram, et eius speciem, id est pulchritudinem, et eius ordinem in ipsum finem. Et haec de secundo articulo. Quantum ad tertium, sciendum est quod ista tria, in quantum sunt partes vestigii, super creaturam, in qua non sunt, non addunt rem aliquam absolutam: ipsa enim essentia creata seipsa limitata est, ita quod ille ablativus non importat habitudinem causae efficientis, sed habitudinem per modum causae formalis, ita quod limitatio creatae essentiae super ipsam creatam essentiam non addit nisi relationem ad causam efficientem, et sua pulchritudo relationem ad causam exemplarem, et eius ordo relationem ad causam finalem, aliter enim esset modus modi, pulchritudo pulchritudinis, ordo ordinis in infinitum, aut non reperiretur in qualibet creatura vestigium Dei. Et sic patet solutio ad quaestionem tertiam".

Robert Cowton, In Sent. I, d. 3, q. 2, (f. 34vb): "De primo, sciendum quod vestigium est impressio facta a pede animalis transeuntis per quam impressionem investigatur via incessus eius non secundum individuum determinatum hoc vel illud, sed secundum genus vel speciem, eo quod non representat hoc animal distincte secundum totum eius, quia non imprimebatur a toto secundum totum eius, sed secundum partem eius. Et quia representatio animalis non secundum determinatam rationem huius individui determinati, sed secundum speciem vel genus est representatio confusa, ideo tractum est nomen illius vestigii ad [fud] similitudinem cuiuslibet rei ipsum confuse et debiliter representantem, quia ergo omnis creatura gerit aliquo modo sue cause similitudinem; et Deus est cuiuslibet creature causa; et ita quelibet creatura gerit debili et confuse aliqua $<\mathrm{m}>$ similitudinem Dei, maxime propter infinitam sublimitatem Dei super creaturam. Ideo quelibet creatura vestigium creatoris portat per quod aliqua cognitio terminorum [de] investigari potest quantum ad essentialia appropriata tribus personis ut patebit statim. Quantum ad secundum articulum dico quod modus uno modo idem est quod terminus et, quia terminatus est limitatum, est et mensuratum. Et ideo accipitur modus interdum pro rei similitudine vel mensura; species autem interdum accipitur pro pulchritudine secundum quod dicitur: 'ille est valde speciosus', id est pulcher. Ex hoc autem quod Deus est causa effectiva creaturarum, exemplaris et finalis oportet in omni creatura esse limitationem eo ipso quod creata est; et pulchritudinem autem eo ipso quod ad pulchrum exemplar formata est a perfectissimo artifice; et ordinem eo ipso quod ad finem <est $>$ : in re omni enim que habet finem oportet ordinem esse ad finem. Quia ergo modus creature, hoc est eius limitatio et mensura, Deum representat sub ratione cause efficientis, pulchritudo vel species sub ratione cause exemplaris, et ordo sub ratione cause finalis, que est representatio indistincta, quia non est quantum ad propria personarum, sed quantum ad appropriata, scilicet sapientiam, et potentiam, et bonitatem; ideo in omni creatura dicitur consistere Dei vestigium quantum ad eius modum, scilicet limitationem vel mensuram, et eius species, id est pulchritudinem, et eius ordinem in ipsum finem, ad hec tria reducuntur omnia alia in quibus ab aliis signatur vestigium. De tertio articulo sciendum quod hec tria (modus, species et ordo) non addunt super rem que est vestigium rem aliquam absolutam: ipsa enim creatura in seipsa est limitata secundum quod proprius principius terminatur et addit unum respectum ad Deum sub ratione cause efficientis; et sua pulchritudo respectum ad causam exemplarem, et eius ordo respectum <ad causam finalem $>$ in infinitum vel non reperietur in qualibet creatura vestigium Dei, cuius oppositum dicit Augustinus, libro I". 


\section{Bibliografía}

\section{Fuentes}

\section{Manuscritos}

» Robertus de Cowton. In Sententiarum. Oxford, Merton College Library Ms. 117, ff. 1-268.

\section{Ediciones}

"Aegidius Aegidius (1699). In primum librum Magistri Sententiarum. Ed. de Aguilar, A. Córdoba.

》 Augustinus Hipponensis (1841). Epistulae. Ed. Migne, J.-P. París. (PL 33).

"Augustinus Hipponensis (1892). De natura boni. Ed. Zycha, J. Viena. (CSEL 25/2).

» Augustinus Hipponensis (1968). De Trinitate. Ed. Mountain, W. Turnhout: Brepols. (CCSL 50A).

" Bonaventura de Balneoregio (1882). Commentarius in I librum Sententiarum (Opera Omnia 1). Ed. Quaracchi: Florencia.

" Henricus Gandavensis (1520). Summa quaestionum ordinarium. Ed. Iodocus Badius Ascensius, (2 vv). Parigi. Reimp. Ed. Buytaert, E. (1953). Nueva York / Lovaina/ París: Franciscan Institute Publications.

» Henricus Gandavensis (1983). Quodlibet IX (Opera Omnia 13). Ed. Macken, R. Lovaina: Leuven University Press.

"Ioannes Duns Scotus (1950-2013). Ordinatio (Opera Omnia 1-14). Ed. Vaticana: Ciudad del Vaticano.

》 Ioannes Duns Scotus (1960-2004). Lectura (Opera Omnia 16-21). Ed. Vaticana: Ciudad del Vaticano.

"Ioannes Duns Scotus (1997). Quaestiones super libros Metaphysicorum Aristotelis. Opera Philosophica 3-4. Ed. Andrews, R. Nueva York: Franciscan Institute Publications.

" Ioannes Duns Scotus (2004). Reportatio I-A. Eds. Wolter, A. y Bychkov, O. Nueva York: Franciscan Institute Publications.

» Petrus Lombardus (1971). Sententiae in IV libris distinctae. Ed. Quaracchi: Grottaferrata.

» Richardus de Mediavilla (1591). Super quatuor libros Sententiarum. Ed. Ludovicus Silvestrius a S. Angelo in Vado: Brescia.

" Thomas Aquinas (1888-1905). Summa Theologica (Opera Omnia 4-12). Ed. Leonina: Roma.

» Thomas Aquinas (1929). Scriptum super libros Sententiarum magistri Petri Lombardi episcopi Parisiensis. Ed. Mandonnet, P. París: Lethielleux. 


\section{Bibliografía complementaria}

» Beckmann, J. P. (1996). "Entdecken oder setzen? Die Besonderheit der Relationstheorie des Duns Scotus und ihre Bedeutung für die Metaphysik". En: Honnefelder, L. et al. (eds.). John Duns Scotus. Metaphysics and Ethics. Nueva York/ Leiden/Colonia: Brill.

" Beierwaltes, W. (1969). "Augustins Interpretation von Sapientia 11, 21", Revue d'études augustiniennes 15, 51-61.

»Decorte, J. (1995). "Modus or res: Scotus's criticism of Henry of Ghent's conception of the reality of a real relation". En: Sileo, L. (ed). In Via Scoti. Methodologica ad mentem Johannis Duns Scoti. Roma: Antonianum.

» Falà, J. F. (2017). Univocità, statuto delle essenze e scienza divina nelle Collationes oxonienses. Un dibattito nell'ordine minorita inglese agli inizi del XIV secolo. PhD Thesis: Macerata.

»Fedeli, M. (2014). Relazione e Trinità nelle Collationes oxonienses attribuite a Giovanni Duns Scoto. PhD Thesis: Macerata.

»Henninger, M. G. (1989). Relations. Medieval theories 1250-1325. Oxford: Oxford University Press.

" Henninger, M. G. (2013). "John Duns Scotus and Peter Auriol on the ontological status of relations", Quaestio 13, 221-242.

» Rosemann, P. W. (2007). The Story of a Great Medieval Book: Peter Lombard's Sentences. Toronto: University of Toronto Press. 